\title{
Mindful Parenting: A Behavioral Tool for Parent Wellbeing
}

Jessica L. Fuller and Elizabeth A. Fitter

The Chicago School of Professional Psychology 


\begin{abstract}
Parents often suffer from conditions such as stress and depression due to the high demands of parenting. In particular, parenting children with some form of developmental disability may bring about increased maladaptive behaviors that may increase daily parenting stressors. The fast-spreading repercussions of the COVID-19 pandemic has left millions of parents across the globe to deal with various stressors in isolation. Recent studies have demonstrated the effectiveness of mindfulness as an intervention on targeting individuals' behaviors such as aggression, SIB, and non-compliance while increasing overall well-being and happiness levels. Significant decreases in target behaviors are noted across studies as well as increases in parent satisfaction and well-being. This study proposes to review the use of mindfulness within the scope of behavior analysis as a tool that can be quickly implemented to support parents not only through this crisis but throughout parenthood in general.
\end{abstract}

Keywords: mindfulness, parent training, COVID-19, behavior reduction, parent wellbeing 


\section{Mindful Parenting: A Behavioral Tool for Parent Wellbeing}

\section{Editor's Note}

This manuscript is being published on an expedited basis, as part of a series of emergency publications designed to help practitioners of applied behavior analysis take immediate action to adjust to and mitigate the COVID-19 pandemic. This article was submitted on 4/10/20 and received final acceptance on 04/27/20. The journal would like to especially thank Amanda Chastain for her expeditious review of the manuscript. The views and strategies suggested by the articles in this series do not represent the positions of the Association for Behavior Analysis, International or Springer Nature.

\section{Introduction}

Over the last two decades, mindfulness has received a great deal of attention going from a mainstream view to scientific investigation across disciplines (Kabat-Zinn \& Davidson, 2011; Adkins et al., 2010; Felver et al., 2017; Ahemaitijiang, Hu, Yang, \& Han, 2020). Fields, such as psychology, have developed the notion that individuals experience significant changes in their quality of life and happiness when a decrease in focus and judgement of private events occur and an increase in awareness of the present moment arises. At the time of publication, a global pandemic (COVID-19) has swiftly altered the way of life for most people around the globe. Many countries have closed their borders, and strict governmental orders have been set in place to ensure the citizens of the world stay safe within their homes and aid in flattening the curve of the rapidly growing cases of people infected. Needless to say, this has swiftly altered the way of life of many parents leaving them to address all daily needs, from education to social skills, within the home setting with little or no support from the outside world. Some countries such as 
the USA have specific guidelines to continue to support parents through telehealth practices, however, many places throughout the world do not count on such support leaving them to deal with parental stress on their own.

Parent stress levels have been associated with an increase in maladaptive behaviors displayed by both typical children and those with some form of disability (Hastings, 2002; Neece et al., 2012; Ó Donnchadha, 2018; Singh et al., 2014e). In addition, behavioral problems tend to be more severe amongst children with autism spectrum disorder (ASD) (Beer, Ward, \& Moar, 2013). Parents not only experience added stress (Cassidy, McConkey, Truesdale-Kennedy, \& Slevin, 2008; Lewis et al., 2006), they experience levels of distress associated with depressive and anxiety symptoms (Beer, Ward, \& Moar, 2013; Benson, 2006), and psychological wellbeing (Lewis et al., 2006; Hastings, 2003). Kabat-Zinn and Zabat-Zinn (1998) suggested mindful parenting carries benefits for both parents and children, regardless of the situation (pg. 49). This may be due to the increase in the parents ability to look at a stressful situation without judgement. Mindful parenting has aided in the decrease of maladptive behaviors by increasing parent attention to the present moment, increasing engagement with the child, and by decreasing judgement of the situation.

\section{Mindfulness}

The term mindfulness has a wide range of meanings and components, but typically includes one or more of the following: meditation practice based on concentration, behavioral practices, and cognitive and empathic strategies (Singh et al., 2008h). Because mindfulness is associated with covert behaviors, it is vital to ensure that clear operational definitions and measurement systems are in place. Bishop et al. (2004) suggested a two-component model to describe mindfulness, which involved a) the self-regulation of attention and b) adopting a 
particular orientation towards one's experience in the present moment. Self-regulation of attention is defined as bringing awareness to the present moment and attending to everything that is happening in both our immediate environment and within our minds (i.e., thoughts, feelings, and sensations). Orientation to one's experience is defined as noticing what one is feeling at that moment without attempting to change it, instead just observing it with curiosity and acceptance.

The development of mindfulness-based approaches is aligned with the history of science and the theory development based on experiential data (Singh et al. 2008h). For example, Langer (1989) presented the theory of mindfulness and its application to human behavior. His research accumulated data of how manipulating our thinking can lead to a better quality of life.

\section{Mindfulness-Based Interventions}

There is a growing body of literature within the field of behavior analysis that has more recently looked at the effects of mindfulness as an intervention to make socially significant changes across diverse populations and behaviors, including those of parents and their children (Singh et al., 2006c; Singh et al., 2006d; Singh et al., 2014e; Singh et al., 2007f). This concept adds value to the idea that mindfulness can help parents focus on what matters, the now, and how being present in a child's life is incredibly valuable, more so during these times of uncertainty in our world. Research has also shown that direct care staff and parents who have undergone training in mindfulness, as a result, can bring about feelings of happiness in individuals with profound intellectual disabilities (Singh et al., 2004g). Research on mindfulness in the behavior analytic research community is still relatively new but see Chapter 20 of Cooper, Heron, and Heward (2020) and Little, Tarbox, and Alzaabi (2020) for behavior perspectives on the behavioral repertoires involved in mindfulness. 
A primary goal of Applied Behavior Analysis (ABA) is to select behaviors to decrease and skills to teach that will profoundly impact that individual and family's quality of life (Luiselli et al., 2017). Various articles address that mindfulness training for parents and caregivers creates a meaningful impact on the quality of life of individuals and their families including the quality of relationship between siblings (Singh et al., 2007f). Mindful parenting has also been successful in decreasing the overall stress levels reported by mothers (Singh et al., 2014e). These studies further support that training parents to engage in mindfulness can help effectively manage some of the challenging behaviors displayed by their children while making a significant impact on their own stress levels and well-being.

Our goal with this paper is to select a few mindfulness based interventions that can be broken into steps in order to decrease response effort by both the practitioner and parent while still acquiring benefits from the intervention. This paper also serves as a quick how-to guide to help ABA practitioners to teach parents to incorporate various informal mindfulness-based interventions into their everyday routines.

\section{Meditation on the Soles of the Feet (SoF)}

The Meditation on the Soles of the feet (SoF) is a mindfulness-based training shown to be effective for parents, caregivers, and children (Ahemaitijiang et al. 2020; Singh et al. 2019a; Adkins et al., 2010). The training achieves mindfulness by directing the individual to focus on the present as opposed to the past by engaging in meditation practices and focusing on a neutral object. Through practice, an individual can divert attention from an aversive setting event that may lead to unwanted behavior, to the present moment that is neutral and holds neither a positive nor a negative valence (Singh et al. 2007b). Table 1 summarizes the procedure that is consistent across the literature with additions to observable, operant behaviors. It is recommended that this 
procedure is introduced to the parent during a training session by prompting them though each step, modeling the behavior, and providing feedback when needed. Feedback should be provided thoughout the practice when needed with some form of social positive praise statement such as "You are doing a really great job at keeping your back straight and your feet grounded on the floor." Once the entirety of the sequence has been reviewed, the practitioner should offer statements pertaining to stimulus-response relations such as "this practice may help you when unwanted changes occur". SoF practice is extensively researched and correlated with a decrease in psychological distress for caregivers and their significant others. With regards to social validity, participants, caregivers, and significant others have rated the SoF practice as acceptable, effective, with no untended effects, and would recommend the practice to others (Singh et al., 2019a).

Table 1

Training Procedure for SoF

1. Sit with a straight spine, without slouching or stretching your shoulders

2. Tilt head slightly forward, with the chin tucked slightly toward the throat

3. Have your eyes slightly open or close them lightly, not squinting

4. Have the tip of your tongue touch the upper palate, near the front of the teeth

5. Have the right hand over the left hand on the lap, thumbs touch each other, or left hand flat on left thigh and right hand flat on right thigh 
6. Move your toes, feel your shoes covering your feet or the texture of your socks, curve of your arch, heels of your feet against the back of your shoes

7. Breathe evenly by inhaling on a 3-4 count second and exhale on a 3-4 count second

8. Focus attention on the flow of your breath

9. When you realize that your mind has wandered away, gently refocus your attention on the flow of your breathing

10. Once you are calm, you can walk away with a smile on your face or respond to the incident with a clear, calm, and concise verbal behavior

\section{Mindful S.T.O.P.}

Mindful S.T.O.P. is a well-known and widely used mindfulness tool used to "check-in" and cultivate mindfulness throughout any point in the day (Phang et al., 2014) and can be a useful stimulus prompt for parents and caregivers. S.T.O.P. should be introduced during parent training by the practitioner. A printout or PDF file should be readily available to serve as a prompt for both the practitioner and the parent. It is suggested that the practitioner provides an overview of the purpose of the mindful S.T.O.P. and review each letter in the acronym by reading them aloud, modeling the behavior, and allowing time for the parent to practice. Feedback should be provided if needed or some form of social positive praise statement such as "I like the way you took your time with taking a slow deep breath." Once all four components have been reviewed, the practitioner should offer supportive statements such as "this may help be a cue for you when you are feeling ." In addition, it is suggested to have the 
practitioner provide a brief review of the importance of antecedent strategies and how a visual cue could help remind them to engage in the S.T.O.P. procedure throughout the day. The last recommendation is that the practitioner should provide examples of where they could place the visual in order for it to be the most effective. This could be in the environments in which they are more likely to experience stress (e.g., playroom, office, kitchen, etc.) or where their child's challenging behaviors are more often happening.

\section{Table 2}

Training Procedure for Mindful S.T.O.P

1. Stop. When you notice that you are feeling stressed, anxious, worried, overwhelmed, and you need a moment: stop.

2. Take a breath. If you find yourself getting lost in thoughts about the future, bring yourself back to the present moment (think about what you are doing at this exact moment). Take a breath, look around, and feel your feet on the ground.

3. Observe what is happening within yourself. What are you feeling? What are you thinking? Acknowledge those feelings, take a deep breath, and come back to the present moment.

4. Proceed with full awareness of yourself and your environment.

\section{Surfing the Urge}

Surfing the urge is an informal mindfulness practice that has been used to decrease aggressive behavior (including verbal aggression) through the practice of self-control (Singh et 
al. 2019). The word urge $(n)$ is related to the idea of having a strong desire or impulse. Parents may often experience the urge to engage in reactive behaviors such as yelling or using language stemming out of frustration when experiencing varying levels of stress. Singh et al. 2019 describes that urges may arise in a progressive manner like wakes in which they increase in intensity then dissipate on their own if the person chooses to not engage with that urge. The authors use a surfboard as a metaphor of how to use the breath to navigate through these urges. This technique can be useful for parents to navigate through stressful instances when they are most likely to engage in instances of aggression (i.e., yelling at their children, saying hurtful words, engaging in physical aggression, etc.). Paying attention to the breath can be used as the metaphorical surfboard to ride the waves of urges without engaging in a reactive response, rather, simply observing each urge as it arises with an open curiosity and a non-judgmental demeanor.

Surfing the urge is a strategy that can also be introduced during parent training and can be coached through during an in vivo observation if the moment arises. Prior to teaching the sequence, the parent should be coached through understanding that urges, within the parenting context, are normal and that anger is a powerful emotion that can cloud the feelings of calmness or awareness. In the study by Singh et al. (2019), a foundation of preliminaries is listed as steps to teach parents about the feeling of anger prior to beginning the Surfing the Urge practice and we highly recommend reviewing those steps prior to teaching the following sequence.

\section{Table 3}

Surfing the Urge Practice 
1. Create a visual of a wave with the reminder "Be mindful of arising urges" or "Be mindful of the wave." This could be placed around the family's home or office as a cue to engage in the practice.

2. When you feel an urge arising, focus on the sensation in your body where the urge is presenting itself (e.g., sensations in the stomach, tightness in the chest, heartbeat speeding, tightness in jaw) and notice its intensity.

3. Acknowledge that the urge is there and observe it with non-judgmental awareness, take a deep breath, and remind yourself that it will disseminate, just as a wave does after it peaks.

4. Bring your attention to your breath.

5. Use the flow of your breath to ride that urge as it rises through you. Picture a surfboard riding a wave in the ocean as it begins to arise, peak with intensity, then dissolve at the end. Do this for a couple of minutes. Suggest setting a timer for 1 or 2 minutes to practice.

6. Shift your attention to where the initial sensation of that urge was present in your body. If it was the tensing of your jaw, does it feel more relaxed? Focus on how riding the wave decreases those sensations associated with the urge.

7. Focus on your breath once again, observe the urge until it is no longer present. 
8. Lastly, show appreciation to yourself for remaining present through the urge without giving into the behaviors you would normally give into. Smile and take a mental note that you responded with awareness and not out of impulsivity.

\section{Self-monitoring}

Behavior analysts have the obligation to collect data and monitor any behavior change procedure in order to make appropriate recommendations. Self-monitoring is a procedure in which an individual collects data on their own behavior (Cooper et al., 2020). Self-monitoring can be a very useful tool for both parents and therapists in order to track progress and monitor the intervention. Creating a simple self-monitoring checklist that tracks the frequency of meditation throughout the day, week, and month could assist the therapist and analyst in tracking behavior change. There are many great applications that could be used in lieu of a traditional data collection sheet and may decrease the response effort of data collection for many parents (i.e., calm, headspace). Consider learning more about these to compliment the valuable use of the interventions listed above.

\section{Discussion}

The studies reviewed for this manuscript made a meaningful impact on the quality of life of individuals and their families. All studies highlighted that mindfulness as an intervention on both caretakers and parents allow them to fully attend, be in the present moment, be less reactive, and engage in more meaningful interactions to make a positive change in others' lives; all qualities that could potentially help alleviate some of the stress related behaviors happening due to our current state of pandemic. The procedures suggested for practice have been adapted from their original versions to facilitate training between practitioner and parent. It is important to note that just like with any other practice, repeated engagement in a behavior will strengthen the 
practice and become a habit. Taking some time out of each day to practice mindfulness will strengthen the practice and will begin to generalize to many situations in everyday life and across different environments. The goal with this paper is to present the groundwork for introducing mindfulness to parents through some simple yet effective mindfulness-based interventions that with consistency and practice will have lasting impacts beyond the current global crisis. 


\section{References}

Adkins, A. D., Singh, A. N., Winton, A. S. W., McKeegan, G. F., \& Singh, J. (2010). Using a mindfulness-based procedure in the community: Translating research to practice. Journal of Child and Family Studies, 19(2), 175-183.

Ahemaitijiang, N., Hu, X., Yang, X., \& Han, Z. R. (2020). Effects of meditation on the soles of the feet on the aggressive and destructive behaviors of chinese adolescents with autism spectrum disorders. Mindfulness, 11(1), 230-240.

Beer, M., Ward, L., \& Moar, K. (2013). The Relationship Between Mindful Parenting and Distress in Parents of Children with an Autism Spectrum Disorder. Mindfulness, 4(2), $102-112$.

Bishop, S. R., Lau, M., Shapiro, S., Carlson, L., Anderson, N. D., \& Carmody, J., et al. (2004). Mindfulness: a proposed operational definition. Clinical Psychology: Science and Practice, 11(3), 230-241.

Cassidy, A., McConkey, R., Truesdale-Kennedy, M., \& Slevin, E. (2008). Preschoolers with autism spectrum disorders: The impact on families and the supports available to them. Early Child Development and Care, 178(2), 115-128.

Cooper, J., Heron, T., \& Heward, W. (2020). Applied behavior analysis (Third ed.). Hoboken, NJ: Pearson Education.

Felver, J. C., Doerner, E., Jones, J., Kaye, N. C., \& Merrell, K. W. (2013). Mindfulness in school psychology: Applications for intervention and professional practice. Psychology in the Schools, 50(6), 531-547.

Hastings, R. (2002). Parental stress and behaviour problems of children with developmental disability. Journal of Intellectual \& Developmental Disability, 27(3), 149-160. 
Kabat-Zinn, J., \& Davidson, R. J. (2011). The mind's own physician: a scientific dialogue with the Dalai Lama on the healing power of meditation. Oakland, CA: New Harbinger.

Kabat-Zinn, M., \& Kabat-Zinn, J. (1998). Everyday blessings: the inner work of mindful parenting. New York: Hachette Books.

Lewis, P., Abbeduto, L., Murphy, M., Richmond, E., Giles, N., Bruno, L., ... Orsmond, G. (2006). Psychological well-being of mothers of youth with fragile X syndrome: Syndrome specificity and within-syndrome variability. Journal of Intellectual Disability Research, 50(12), 894-904.

Little, A., Tarbox, J., \& Alzaabi, K. (2020). Using Acceptance and Commitment Training to enhance the effectiveness of behavioral skills training. Journal of Contextual Behavioral Science. DOI: 10.1016/j.jcbs.2020.02.002.

Luiselli, K., Worthen, D., Carbonell, L., \& Queen, H. (2017). Social Validity Assessment of Mindfulness Education and Practices Among High School Students. Journal of Applied School Psychology, 33(2), 124-135. https://doi.org/10.1080/15377903.2016.1264531

Neece, C., Green, S., \& Baker, B. (2012). Parenting stress and child behavior problems: A transactional relationship across time. American Journal on Intellectual and Developmental Disabilities, 117(1), 48-66.

Ó Donnchadha, S. (2018). Stress in caregivers of individuals with intellectual or developmental disabilities: A systematic review of mindfulness-based interventions. Journal of Applied Research in Intellectual Disabilities : Jarid, 31(2), 181-192. doi:10.1111/jar.12398

Phang, C., Keng, S., \& Chiang, K. (2014). Mindful-s.t.o.p.: Mindfulness made easy for stress reduction in medical students. Education in Medicine Journal, 6(2). 
Singh, N., Lancioni, G., Karazsia, B., Myers, R., Kim, E., Chan, J., . . Janson, M. (2019). Surfing the urge: An informal mindfulness practice for the self-management of aggression by adolescents with autism spectrum disorder. Journal of Contextual Behavioral Science, 12, 170-177. doi:10.1016/j.jcbs.2018.10.003

Singh, N. N., Lancioni, G. E., Medvedev, O. N., Sreenivas, S., Myers, R. E., \& Hwang, Y. S. (2019a). Meditation on the soles of the feet practice provides some control of aggression for individuals with Alzheimer's disease. Mindfulness, 10(7), 1232-1242.

Singh, N. N., Lancioni, G. E., Winton, A. S. W., Adkins, A. D., Singh, J., \& Singh, A. N. (2007b). Mindfulness training assists individuals with moderate mental retardation to maintain their community placements. Behavior Modification, 31(6), 800-814.

Singh, N., Lancioni, G., Winton, A., Curtis, W., Wahler, R., Sabaawi, M., McAleavey, K. (2006c). Mindful staff increase learning and reduce aggression in adults with developmental disabilities. Research in Developmental Disabilities, 27(5), 545-558.

Singh, N., Lancioni, G., Winton, A., Fisher, B., Wahler, R., Mcaleavey, K., Sabaawi, M. (2006d). Mindful parenting decreases aggression, noncompliance, and self-injury in children with autism. Journal of Emotional and Behavioral Disorders, 14(3), 169-177. doi:10.1016/j.ridd.2005.07.002

Singh, N. N., Lancioni, G. E., Winton, A. S., Karazsia, B. T., Myers, R. E., Latham, L. L., \& Singh, J. (2014e). Mindfulness-based positive behavior support (MBPBS) for mothers of adolescents with autism spectrum disorder: Effects on adolescents' behavior and parental stress. Mindfulness, 5(6), 646-657.

Singh, N. N., Lancioni, G. E., Winton, A. S., Singh, J., Curtis, W. J., Wahler, R. G., \& McAleavey, K. M. (2007f). Mindful parenting decreases aggression and increases social 
behavior in children with developmental disabilities. Behavior Modification, 31(6), 749771

Singh, N. N., Lancioni, G. E., Winton, A. S., Wahler, R. G., Singh, J., \& Sage, M. (2004g). Mindful caregiving increases happiness among individuals with profound multiple disabilities. Research in Developmental Disabilities, 25(2), 207-218.

Singh, N. N., Lancioni, G. E., Wahler, R. G., Winton, A. S. W., \& Singh, J. (2008h). Mindfulness approaches in cognitive behavior therapy. Behavioral and Cognitive Psychotherapy, 36(6), 659-666

Singh, N.N., Singh, A.N., Lancioni, G.E., Singh, J., Winton, A.S.W., \& Adkins, A.D. (2009i). Mindfulness training for parents and their children with ADHD increases the children's compliance. Journal of Child and Family Studies, 19(2), 157-166. 